\title{
Olfactory Nerve Disorder, CTCAE
}

National Cancer Institute

\section{Source}

National Cancer Institute. Olfactory Nerve Disorder, CT CAE. NCI Thesaurus. Code C143712.

A disorder characterized by dysfunction of the olfactory nerve (first cranial nerve). 